\title{
Caracterización morfológica de semillas de frijol caupí (Vigna unguiculata L. Walp) de la Península de Yucatán
}

\section{Morphological characterization of cowpea beans (Vigna unguiculata L. Walp) from the Yucatán Peninsula}

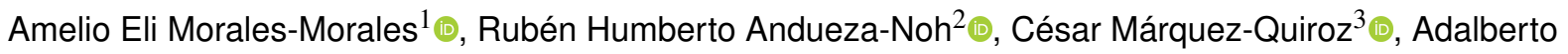 \\ Benavides-Mendoza ${ }^{4}$ (D), José María Tun-Suarez ${ }^{1}$ (D), Alejandra González-Moreno ${ }^{1}$ (), Carlos Juan \\ Alvarado-López ${ }^{2 *}$ (1) \\ ${ }^{1}$ División de Estudios de Posgrado e Investigación, Tecnológico Nacional de México / Instituto Tecnológico de Conkal. Av. Tecnológico \\ s/n. Conkal, Yucatán, México. \\ ${ }^{2}$ Conacyt - Instituto Tecnológico de Conkal. Av. Tecnológico s/n, CP. 97345. Conkal, Yucatán, México. \\ ${ }^{3}$ Posgrado en Ciencias Agroalimentarias. División Académica de Ciencias Agropecuarias, Universidad Juárez Autónoma de Tabasco, \\ Carretera Villahermosa-Teapa km 25 R/a La Huasteca $2^{a}$ sección, CP. 86280. Villahermosa, Centro, Tabasco, México. \\ ${ }^{4}$ Departamento de Horticultura, Universidad Autónoma Agraria Antonio Narro, Calz Antonio Narro 1923, CP. 25315. Buenavista, Saltillo, \\ Coahuila, México. \\ *Autor de correspondencia: carlos.alvarado@itconkal.edu.mx
}

Artículo científico recibido: 07 de marzo de 2019 aceptado: 06 de junio de 2019

RESUMEN. El frijol caupí (Vigna unguiculata (L) Walp) es un cultivo importante en varias regiones del mundo, pero en México no es muy explotado. La caracterización de variedades agrícolas se ha basado en descriptores morfológicos, en los que se incluyen: forma, color y tamaño de semilla, además de atributos cuantitativos como el contenido mineral. El objetivo del trabajo fue evaluar las características morfológicas y nutricionales de genotipos de frijol caupí de la Península de Yucatán, México. Se realizaron 15 colectas de octubre 2017 a enero 2018, a las que se evaluaron tres variables morfológicas cualitativas, 10 variables cuantitativas y se determinó el contenido de potasio, calcio, manganeso, hierro y zinc, mediante $\mu$-fluorescencia de rayos-X. A los datos se les realizó un análisis de varianza y de componentes principales. El $80 \%$ de las colectas tienen forma ovoide, $73 \%$ textura suave a áspera y el $80 \%$ son de color negro. En las variables morfológicas cuantitativas, $53 \%$ de las colectas se consideran pequeñas, $40 \%$ medianas y $7 \%$ semillas grandes. La longitud, ancho y espesor de los genotipos fue de 6.26 a 9.56, 3.64 a 7.48 y de 2.86 a $5.56 \mathrm{~mm}$, respectivamente, la colecta PET15 fue la de menor dimensión. Para el contenido mineral, la colecta OXC04 tuvo la mayor concentración de $\mathrm{Ca}, \mathrm{Mn}$, Fe y Zn, mientras que la colecta PET07 presentó mayor contenido de K. Hay altos niveles de diversidad fenotípica en las colectas estudiadas y se agrupan con base en sus características físicas y no al lugar de origen.

Palabras clave: Colecta, contenido mineral, diversidad fenotípica, fluorescencia de rayos- $\mathrm{X}$, genotipos.

ABSTRACT. The cowpea bean (Vigna unguiculata $(\mathrm{L})$ Walp) is an important crop in several regions of the world; however, in Mexico it is not very exploited. The characterization of agricultural varieties has traditionally been based on morphological descriptors, which include: shape, color and size of the seed, in addition to quantitative attributes such as mineral content. The objective of the work was to evaluate the morphological and nutritional characteristics of the bean genotypes of the Yucatán Peninsula, Mexico. 15 collections were obtained from October 2017 to January 2018, in which three qualitative morphological variables are evaluated, 10 quantitative variables and five minerals are determined by $\mu$-X-ray fluorescence. Data were analyzed for variance and principal component analysis. The $80 \%$ of the collectivities are ovoid, $73 \%$ have a smooth texture and $80 \%$ have a black color. In the quantitative morphological variables, $53 \%$ of the collective ones are small, $40 \%$ are medium sized and 7\% are large seeds. The length, width and thickness of the genotypes were from 6.26 to $9.56,3.64$ to 7.48 and from 2.86 to $5.56 \mathrm{~mm}$, respectively, the PET15 collection was the smallest. Regarding the mineral content, the OXC04 collection obtained a higher concentration of $\mathrm{Ca}, \mathrm{Mn}, \mathrm{Fe}$ and $\mathrm{Zn}$, while the PET07 collection presented the highest 
content of K. It is concluded that there are high levels of phenotypic diversity in the collections studied and that they are grouped based on the physical characteristics and not on its geographical origin.

Key words: Collection, mineral content, phenotypic diversity, X-ray fluorescence, genotypes.

\section{INTRODUCCIÓN}

El género Vigna pertenece a la familia Fabaceae, que comprende más de 200 especies distribuidas en el mundo, de las cuales las de mayor importancia agrícola son $V$. radiata, $V$. mungo, $V$. angularis, $V$. subterranea, $V$. aconitifolia, $V$. umbellata y $V$. unguiculata (Fery 2002). Esta última especie a nivel mundial se conoce como frijol caupí, y su centro de origen es África, aunque se desconoce la región exacta (Xiong et al. 2016, Carvalho et al. 2017). Se cultiva principalmente en regiones tropicales y subtropicales del mundo (Boukar et al. 2013), por lo que es una de las cinco leguminosas más importantes para consumo humano (Smýkal et al. 2015). Es una especie tolerante a la sequía, presenta buena capacidad de fijación de nitrógeno y se adapta a diferentes sistemas de cultivo (Goncalves et al. 2016), además es rico en proteínas y carbohidratos (Menssen et al. 2017), por lo que es un cultivo económicamente rentable para los pequeños agricultores (Deng et al. 2013, Segura-Campos et al. 2013).

En México, se conoce como frijol pelón o xpelón en el sureste del país, mientras que en el centro y norte se conoce como chicharro de vaca, frijol chino o frijol yorimón (Lagunes-Espinoza et al. 2008). Se cultiva principalmente bajo el sistema de agricultura de temporal y en la península de Yucatán, se produce bajo cultivo convencional, así como, en la milpa tradicional (Arias et al. 2004, SIAP 2017). Es una fuente importante de ingresos económicos para los campesinos, además de proporcionar los elementos nutricionales esenciales para la población local que lo consume en diferentes platillos típicos de la región (García-Hernández et al. 2005, Márquez-Quiroz et al. 2015).

La caracterización de semillas se basa en el empleo de descriptores morfológicos o fenotípicos, como la forma, el color y tamaño de la semilla; pero atributos cuantitativos como el contenido mineral, son características de interés en especies como el frijol caupí que está bajo creciente demanda en el mercado (Stoilova y Pereira 2013, Wamalwa et al. 2016). Al respecto Coulibaly y Lowenberg-DeBoer (2002) mencionan que la calidad de las semillas se relaciona con las características morfológicas, como el tamaño, el color del micrópilo, el grosor y la textura de la testa. Los estudios de caracterización morfológica de frijol caupí se han realizado principalmente en África y Sudamérica, reportando una gran diversidad genética. Al respecto, Ghalmi et al. (2010) identificaron variabilidad en color de grano, e identificaron que el mayor porcentaje de granos (72\%) tenían textura suave. Sobre lo mismo Mafakheri et al. (2017) al evaluar diferentes genotipos reportan que el tamaño de la semilla (longitud, ancho y espesor) varío de 6.74 a $9.44,5.69$ a 7.63 y de 4.21 a $5.70 \mathrm{~mm}$, respectivamente. Mientras que Menssen et al. (2017) reportan que el peso de 100 semillas oscila entre 7.67 y 15.12 g.

Los estudios de diversidad morfológica y mineral del frijol caupí en México son escasos, por lo que no se tienen datos sobre la diversidad de esta especie en el país, aunque se sabe que los agricultores seleccionan la semilla de sus cosechas prefiriendo las variedades criollas (Latournerie-Moreno et al. 2006). Pero esto puede afectar la diversidad genética del frijol caupí en la región, debido a la falta de conocimiento científico para conservar el recurso genético y por la pérdida del conocimiento ancestral (Vara-Sánchez y Padilla 2013). Por lo anterior, el objetivo del estudio fue evaluar las características morfológicas y el contenido mineral de los genotipos locales de frijol caupí cultivados en la península de Yucatán, para su aprovechamiento en el mejoramiento genético, manejo y conservación.

\section{MATERIALES Y MÉTODOS}

\section{Colecta}

Las colectas de frijol caupí se realizaron de octubre de 2017 a enero de 2018, en siete municipios de 
la península de Yucatán. Se obtuvieron 15 colectas de semillas con los productores en sus áreas de cultivo, milpas y traspatios; de las cuales dos provienen de municipios del estado de Campeche, tres del estado de Quintana Roo y 10 del estado de Yucatán (Tabla 1).

\section{Caracterización morfológica}

Se evaluaron 13 variables, tres cualitativas y 10 cuantitativas. Las variables cualitativas se midieron con base en el descriptor del IBPGR (1983), para la variable forma de la semilla se consideraron las categorías: 1 = riñón, 2 = ovoide, 3 = cuboide, $4=$ globosa, 5 = romboides; para la textura de la testa se consideraron las categorías 1 = suave, 2 = suave a áspera, 3 = rugosa; y para el color de la semilla se consideraron las categorías $1=$ crema, $2=$ crema marrón, $3=$ marrón, $4=$ marrón ocre, $5=$ marrón oliva, $6=$ blanco y negro, $7=$ blanco $8=$ negro y 9 $=$ rojo.

Las variables cuantitativas fueron: peso de la semilla, y peso de 100 semillas, por triplicado con una balanza portátil marca POKET SCALE modelo $\mathrm{MH}$-series. Longitud de semilla, ancho y espesor, se midió en 10 semillas tomadas al azar de cada colecta con un vernier digital marca Truper Staninless steel ${ }^{\circledR}$ con precisión $\pm 0.1 \mathrm{~mm}$, de acuerdo con la metodología propuesta por Demooy y Demooy (1990). Con las mediciones de las semillas, se calcularon las siguientes variables: relación entre ancho y longitud de la semilla, diámetro medio aritmético, diámetro medio geométrico, y esfericidad con las ecuaciones descritas por Altuntaş y Yıldız (2007) y Cetin (2007).

El grosor de la testa se determinó en 10 semillas tomadas al azar, colocando cada semilla en un portaobjeto de vidrio y se desgastó con lijas de agua número 400 , hasta llegar al plano mediano el cual permitió hacer la medición con un microscopio digital (Dino-Lite 2.0).

\section{Determinación del contenido mineral}

El análisis de contenido mineral se realizó por $\mu$-fluorescencia de rayos- $X$ de energía dispersiva ( $\mu$ EDXRF) (M4 TornadoTM), con la técnica utilizada por
Cardoso et al. (2018). El generador de rayos $X$ para las mediciones de todos los elementos se operó a 50 $\mathrm{kV}$ y $100 \mu \mathrm{A}$ con un filtro de 12.5A. La detección de la radiación de fluorescencia se realizó mediante un detector de dispersión de silicio, dispersor de energía XFlashTM, con un área sensible de $30 \mathrm{~mm}^{2}$ y resolución de energía de $142 \mathrm{eV}$ para todos los elementos. Se cuantifico el potasio $(\mathrm{K})$, calcio $(\mathrm{Ca})$, manganeso $(\mathrm{Mn})$, hierro $(\mathrm{Fe})$ y zinc $(\mathrm{Zn})$ en $\mathrm{mg} \mathrm{kg}^{-1}$, principalmente por el aporte nutricional que estos proveen a la población.

\section{Análisis de datos}

Los datos de las variables cualitativas se analizaron con estadística descriptiva transformando los rangos observados de cada categoría en porcentajes. Mientras que los datos obtenidos de las variables morfológicas cuantitativas y al análisis mineral se les aplicó análisis de varianza, para luego realizar una comparación de medias con la prueba de diferencia mínima significativa (LSD) con 95\% de confiabilidad a las variables que tuvieron diferencias significativas con el software estadístico SAS 9.2 (SAS 2009).

Para evaluar las relaciones entre las colectas, se utilizaron las 13 variables morfológicas estandarizadas a $\mu=0$ y $\sigma^{2}=1$, para realizar el análisis de componentes principales (ACP) con el criterio de rotación varimax, utilizando la matriz de correlaciones mediante el programa estadístico SPSS (SPSS 2016). Se utilizó la regla de Kaiser (1960) para determinar la significancia de los eigenvalores y eigenvectores de cada componente. Además, se realizó un análisis de conglomerados mediante el método de UPGMA con la distancia Euclidiana como medida de similitud.

\section{RESULTADOS}

\section{Diversidad fenotípica}

Los resultados obtenidos con las variables cualitativas (Tabla 2), muestran que el $80.0 \%$ de las colectas tienen semillas de forma ovoide, $13.3 \%$ globosa y $6.7 \%$ romboidal. Para la textura de la testa, el $73.3 \%$ de las colectas tienen testa con textura de 
Morales-Morales et al.

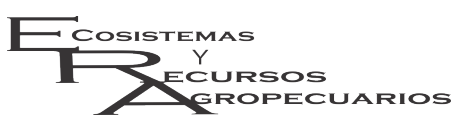

Caracterización de semillas de caupí

Ecosist. Recur. Agropec. 6(18):463-475,2019

Tabla 1. Sitios de colecta del frijol caupí en la península de Yucatán.

\begin{tabular}{|c|c|c|c|c|c|c|}
\hline $\begin{array}{c}\text { Clave } \\
\text { colecta }\end{array}$ & $\begin{array}{c}\text { Nombre } \\
\text { común }\end{array}$ & Localidad & Municipio & Estado & $\begin{array}{c}\text { Altura } \\
\text { (msnm) }\end{array}$ & Coordenadas \\
\hline OXC01 & Yax pelón & Xul & Oxcutzcab & Yucatán & 89 & $20^{\circ} 06^{\prime} 30.7^{\prime \prime} \mathrm{N} 89^{\circ} 27^{\prime} 35.7^{\prime \prime} \mathrm{W}$ \\
\hline HEC02 & Xpelón & San Vicente Cumpich & Hecelchakán & Campeche & 39 & $20^{\circ} 11^{\prime} 14.5^{\prime \prime} \mathrm{N} 89^{\circ} 58^{\prime} 23.1^{\prime \prime} \mathrm{W}$ \\
\hline HEC03 & Chalack simin & San Vicente Cumpich & Hecelchakán & Campeche & 39 & $20^{\circ} 11^{\prime} 08.6^{\prime \prime} \mathrm{N} 89^{\circ} 58^{\prime} 26.8^{\prime \prime} \mathrm{W}$ \\
\hline OXC04 & Paysin & Xul & Oxcutzcab & Yucatán & 39 & $20^{\circ} 05^{\prime} 41.5^{\prime \prime} \mathrm{N} 89^{\circ} 27^{\prime} 33.5^{\prime \prime} \mathrm{W}$ \\
\hline OxC05 & Paysin & Xul & Oxcutzcab & Yucatán & 89 & $20^{\circ} 06^{\prime} 10.8^{\prime \prime} \mathrm{N} 89^{\circ} 27^{\prime} 38.8^{\prime \prime} \mathrm{W}$ \\
\hline CHE06 & Espelón perón & Kuxeb & Chemax & Yucatán & 25 & $20^{\circ} 51^{\prime} 31.1^{\prime \prime} \mathrm{N} 87^{\circ} 52^{\prime} 05.9^{\prime \prime} \mathrm{W}$ \\
\hline PET07 & Espelón Domingo & Peto & Peto & Yucatán & 35 & $20^{\circ} 07^{\prime} 45.4^{\prime \prime} \mathrm{N} 88^{\circ} 54^{\prime} 22.0^{\prime \prime} \mathrm{W}$ \\
\hline PET08 & Espelón blanco & Peto & Peto & Yucatán & 35 & $20^{\circ} 07^{\prime} 49.5^{\prime \prime} \mathrm{N} 88^{\circ} 54^{\prime} 32.2^{\prime \prime} \mathrm{W}$ \\
\hline JMM09 & Chalack simin negro & San Felipe 1 & José María Morelos & Quintana Roo & 35 & $19^{\circ} 48^{\prime} 45.9^{\prime \prime} \mathrm{N} 88^{\circ} 49^{\prime} 55.2^{\prime \prime} \mathrm{W}$ \\
\hline JMM10 & Espelón & López Mateos & José María Morelos & Quintana Roo & 35 & $19^{\circ} 37^{\prime} 55.9^{\prime \prime} \mathrm{N} 88^{\circ} 41^{\prime} 17.8^{\prime \prime} \mathrm{W}$ \\
\hline PET11 & Xnuuc Pelón & Xoy & Peto & Yucatán & 16 & $20^{\circ} 07^{\prime} 16.2^{\prime \prime} \mathrm{N} 88^{\circ} 58^{\prime} 04.8^{\prime \prime} \mathrm{W}$ \\
\hline CHE12 & Espelón de guía & Mucel & Chemax & Yucatán & 25 & $20^{\circ} 53^{\prime} 22.4^{\prime \prime} \mathrm{N} 87^{\circ} 49^{\prime} 42.9^{\prime \prime} \mathrm{W}$ \\
\hline FCP13 & Espelón & Polyuc & Felipe Carrillo Puerto & Quintana Roo & 20 & $19^{\circ} 36^{\prime} 37.3^{\prime \prime} \mathrm{N} 88^{\circ} 33^{\prime} 27.2^{\prime \prime} \mathrm{W}$ \\
\hline HAL14 & Paysin & Halachó & Halachó & Yucatán & 16 & $20^{\circ} 29^{\prime} 23.8^{\prime \prime} \mathrm{N} 90^{\circ} 05^{\prime} 16.1$ 'W \\
\hline PET15 & Frijol chino & Peto & Peto & Yucatán & 35 & $20^{\circ} 07^{\prime} 45.4^{\prime \prime} \mathrm{N} 88^{\circ} 54^{\prime} 22.0^{\prime \prime} \mathrm{W}$ \\
\hline
\end{tabular}

suave a áspera, mientras que $26.7 \%$ restante tienen textura suave. Para el color de las semillas predominó el color negro (80\%), seguido del blanco $(6.6 \%)$, crema $(6.6 \%)$ y rojo $(6.6 \%)$.

Tabla 2. Características cualitativas de las semillas de frijol caupí.

\begin{tabular}{lccc}
\hline Colecta & Forma & Textura & Color \\
\hline OXC01 & Ovoide & Suave a áspera & Negra \\
HEC02 & Ovoide & Suave & Negra \\
HEC03 & Globosa & Suave & Crema \\
OXC04 & Ovoide & Suave a áspera & Negra \\
OXC05 & Ovoide & Suave a áspera & Negra \\
CHE06 & Ovoide & Suave a áspera & Negra \\
PET07 & Ovoide & Suave a áspera & Negra \\
PET08 & Globosa & Suave a áspera & Blanca \\
JMM09 & Ovoide & Suave a áspera & Negra \\
JMM10 & Ovoide & Suave a áspera & Negra \\
PET11 & Ovoide & Suave a áspera & Negra \\
CHE12 & Ovoide & Suave a áspera & Negra \\
FCP13 & Ovoide & Suave a áspera & Negra \\
HAL14 & Ovoide & Suave & Negra \\
PET15 & Romboide & Suave & Roja \\
\hline
\end{tabular}

Para las variables cuantitativas, se observaron diferencias altamente significativas (Tabla 3). El peso de la semilla y peso de 100 semillas varió de 0.06 a 0.23 y de 3.46 a $22.08 \mathrm{~g}$, respectivamente. El valor mínimo lo tuvo la colecta PET15 y el valor máximo la colecta HEC03. La longitud de semilla fue de 6.26 a $9.56 \mathrm{~mm}$ con promedio de $8.49 \mathrm{~mm}$, presentando la colecta PET15 el valor mínimo, mientras que la colecta JMM09 tuvo el máximo valor. El ancho y espesor de la semilla osciló entre 3.64 y 7.40 y de 2.86 a $5.56 \mathrm{~mm}$, con valores promedios de 6.37 y 4.57 $\mathrm{mm}$, respectivamente. La colecta con menor anchura y espesor de semilla fue la PET15, mientras que el mayor tamaño lo tuvo la HEC03. El diámetro medio aritmético y diámetro medio geométrico fue de 4.25 a 7.50 y de 4.03 a $7.32 \mathrm{~mm}$, respectivamente, mientras que la esfericidad fue de 0.56 a $0.79 \%$. Para el grosor de testa, las colectas PET15 y HEC03 tuvieron la testa más gruesa con valores de 0.18 y $0.15 \mathrm{~mm}$, respectivamente; mientras que las colectas $\mathrm{CHE} 12 \mathrm{y}$ JMM10 tuvieron la testa más delgada.

El análisis de componentes principales, determinó que con los tres primeros componentes principales se explica el $90.53 \%$ de la variación total observada. El CP1 explicó el $61.76 \%$ de la variación total, siendo el peso de la semilla, peso de 100 semillas, la longitud, ancho y espesor de la semilla, diámetro medio aritmético y diámetro medio geométrico, las de mayor contribución. El CP2 explicó el 18.65\% de variación total, siendo las variables cualitativas (forma, textura y color) y grosor de testa las que contribuyeron a la variación explicada de este componente. En tanto que el CP3 explicó el $10.12 \%$ de la variación total con las variables de mayor contribución esfericidad y relación ancho entre longitud de semillas (Tabla 4).

En la Figura 1, se observa la dispersión de las colectas sobre el plano determinado por los dos primeros componentes principales e indica la formación de cuatro grupos con $80.41 \%$ de la variación to- 
Morales-Morales et al.

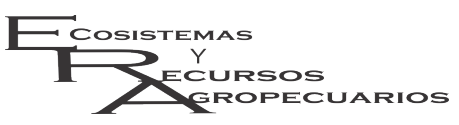

Caracterización de semillas de caupí

Ecosist. Recur. Agropec.

6(18):463-475,2019

Tabla 3. Comparación de medias de las variables cuantitativas de las semillas de frijol caupí.

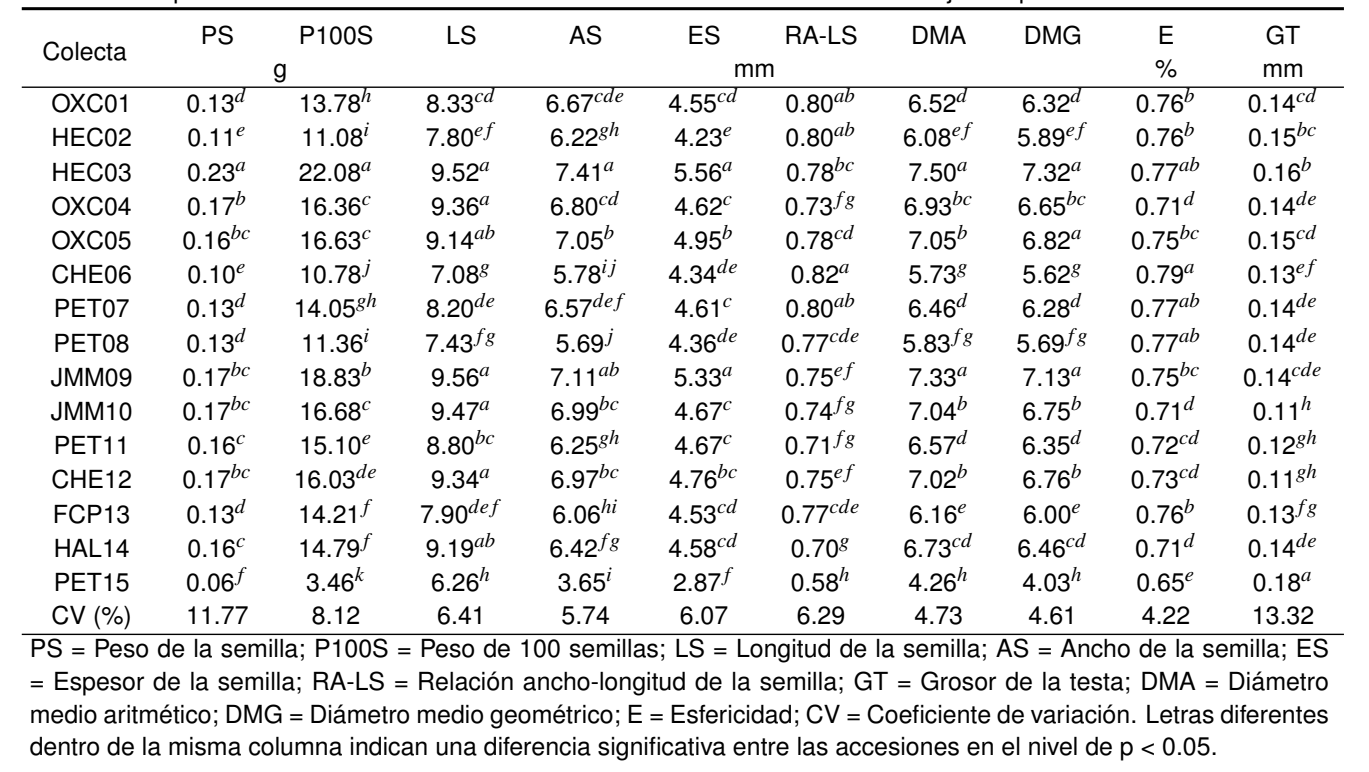

Tabla 4. Vectores propios de los tres primeros componentes principales medidos en 15 accesiones de frijol caupí de la península de Yucatán.

\begin{tabular}{cccc}
\hline Variables & \multicolumn{3}{c}{ Componentes } \\
& 1 & 2 & 3 \\
\hline FS & -0.309 & $-0.734^{*}$ & -0.193 \\
TS & 0.039 & $0.825^{*}$ & 0.169 \\
CS & -0.136 & $-0.728^{*}$ & -0.460 \\
PS & $0.969^{*}$ & 0.046 & 0.058 \\
P100S & $0.963^{*}$ & 0.132 & 0.188 \\
LS & $0.956^{*}$ & 0.235 & -0.132 \\
AS & $0.890^{*}$ & 0.295 & 0.308 \\
ES & $0.891^{*}$ & 0.169 & 0.382 \\
GT & -0.288 & $-0.871^{*}$ & -0.016 \\
RA-LS & 0.236 & 0.297 & $0.904^{*}$ \\
DMA & $0.953^{*}$ & 0.251 & 0.152 \\
DMG & $0.945^{*}$ & 0.246 & 0.206 \\
E & 0.149 & 0.184 & $0.960^{*}$ \\
Variación explicada & $61.76 \%$ & 18.65 & $10.12 \%$ \\
Variación acumulada & $61.76 \%$ & $80.41 \%$ & 90.53 \\
\hline Los * en las columnas significan las variables de mayor \\
contribución para cada componente principal. FS $=$ \\
Forma de semilla; TS = Textura testa; CS = Color de \\
semillas; PS = Peso de semilla; P100S = Peso de 100 \\
semillas; LS = Longitud de semilla; AS = Ancho semilla; \\
ES = Espesor de semillas; GT = grosor de testa; RA-LS \\
= Relación ancho entre longitud semilla; DMA = Diámetro \\
medio aritmético; DMG = Diámetro medio geométrico; E \\
= Esfericidad.
\end{tabular}

tal explicada. Este valor indica que la representación gráfica de los primeros dos componentes principales es apropiada para visualizar la variación morfológica entre los grupos formados y al interior de cada grupo.
El primer grupo (I) se conformó por la colecta PET15, que tiene características relacionadas con la esfericidad y la relación entre el ancho y longitud, peso de 100 semillas. El segundo grupo (II) se formó por las colectas OXC01, OXC04, OXC05, PET07, JMM09, JMM10, PET11, CHE12, FCP13 y HAL14 que presentaron características similares en longitud, anchura y espesor de la semilla. Mientras que el tercer grupo (III) se conformó por las colectas PET08, HEC02 y CHE06 que tienen características similares en longitud de la semilla y esfericidad. El grupo cuatro (IV) lo formó la colecta HEC03 diferenciado por el color, peso de 100 semillas, diámetro medio aritmético y diámetro medio geométrico.

El análisis de conglomerados (Figura 2) construido con el método de UPGMA y la distancia Euclidiana dio soporte a lo observado en el ACP (Figura 1), al identificar cuatro grupos definidos con un coeficiente de similitud de 4.3. La asociación entre las colectas inició con un coeficiente de similitud de 0.30 unidades y se intensificó de forma rápida para dejar diferenciados cuatro grupos a un coeficiente de similitud de 4.3 unidades de las aproximadamente 13.4 unidades.

El grupo I se formó por la colecta PET15 que tiene semillas de color rojo, de forma romboide, pe- 


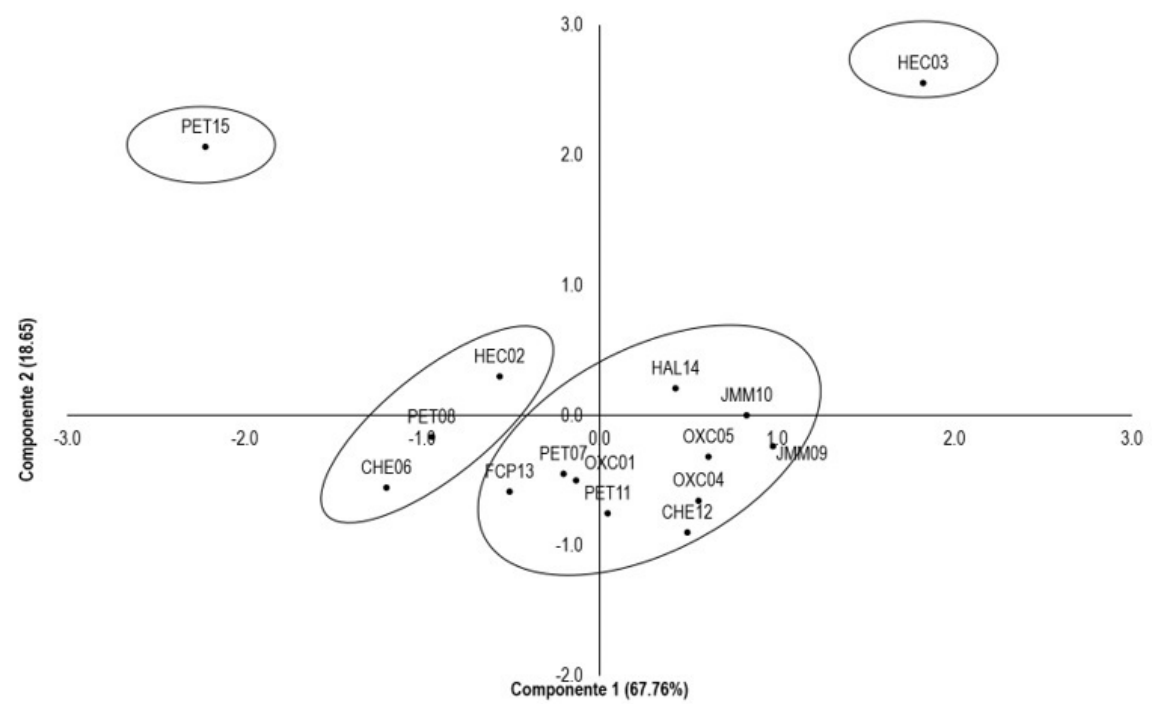

Figura 1. Distribución espacial de las 15 colectas de frijol caupí obtenidos en la península de Yucatán con base a los dos primeros componentes principales y 13 variables morfológicas.

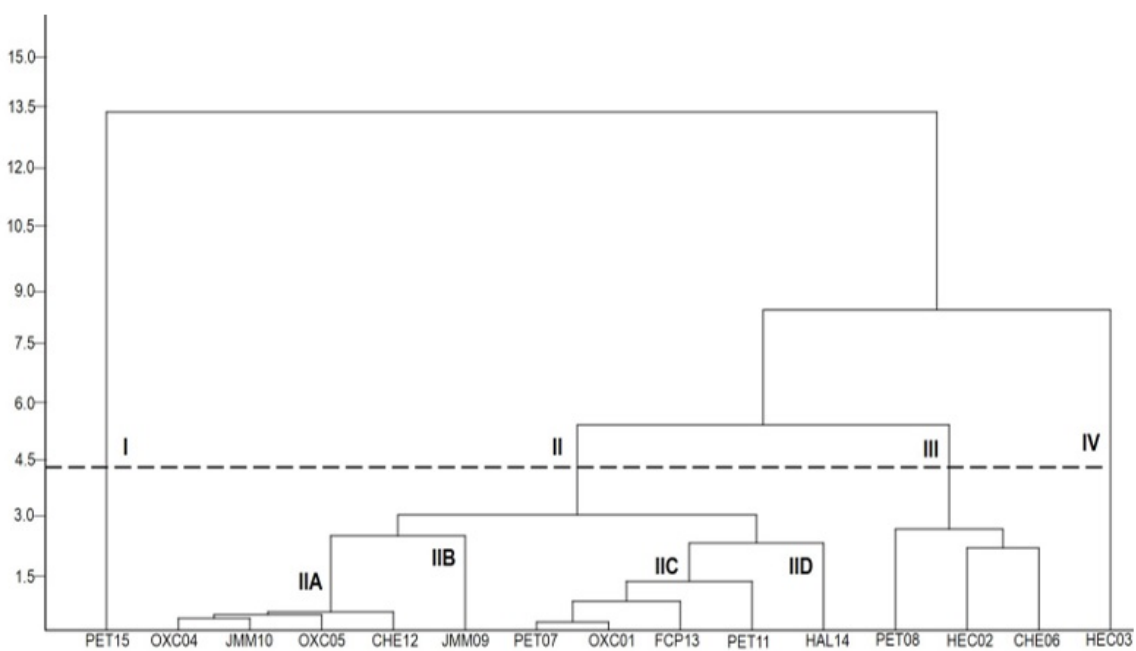

Figura 2. Análisis de conglomerado de las 15 colectas de frijol caupí de la península de Yucatán con base al análisis de dispersión por distancia euclidiana mediante el método de UPGMA con las variables morfológicas.

queñas y testa gruesa. En el grupo dos está formado por diez colectas y se divide en cuatro subgrupos. Este grupo tiene colectas con semillas de forma ovoide, de color negro y textura de suave a áspera. En el subgrupo IIA formado por las colectas OXC04, JMM10, OXC05 y CHE12 que presentan similitud de 0.8 unidades, se caracteriza por tener semillas medianas, con el mayor peso de semilla individual y menor grosor de testa. El subgrupo IIB se formó por la colecta JMM09 que se caracteriza por tener las semillas de mayor longitud, mayor anchura y espesor, además es una colecta con semilla de tamaño grande. El subgrupo IIC se forma por las colectas PET07, OXC01, FCP13 y PET11, se caracteriza por tener semillas pequeñas de color negro, mientras que el subgrupo IID está formado por la colecta HAL14 
que es el único material de color negro que presenta semillas de textura suave. El grupo III está formado por tres colectas PET08, HEC02 y CHE06 que son similares por presentar semillas con longitud, grosor, anchura, espesor y peso de 100 semillas. El grupo IV está formado por la colecta HEC03 que se distingue por tener la semilla de mayor tamaño y de color crema.

Tabla 5. Contenido mineral de frijol caupí colectado en la península de Yucatán.

\begin{tabular}{|c|c|c|c|c|c|}
\hline Colecta & \multicolumn{2}{|c|}{$\mathrm{mg} \mathrm{kg}^{-1}$} & $\mathrm{Mn}$ & $\begin{array}{c}\mathrm{Fe} \\
\mathrm{mg} \mathrm{kg}^{-1}\end{array}$ & $\mathrm{Zn}$ \\
\hline OXC01 & $562.5^{d e}$ & $289.8^{b c d}$ & $3.6^{\text {cde }}$ & $20.3^{b c d}$ & $11.2^{b c d}$ \\
\hline HEC02 & $486.2^{\text {def }}$ & $172.1^{f g}$ & $4.2^{b c}$ & $18.3^{c d e}$ & $7.0^{f g h}$ \\
\hline HEC03 & $616.8^{b c}$ & $207.7^{d e f}$ & $3.9^{b c d}$ & $14.3^{d e}$ & $12.9^{a b}$ \\
\hline OXC04 & $753.5^{b c}$ & $468.3^{a}$ & $8.9^{a}$ & $34.5^{a}$ & $15.0^{a}$ \\
\hline OXC05 & $999.6^{a}$ & $293.7^{b c}$ & $3.8^{\text {cde }}$ & $27.5^{a b}$ & $11.5^{b c}$ \\
\hline CHE06 & $369.1^{f g}$ & $176.1^{f g}$ & $2.5^{d e}$ & $11.8^{e f}$ & $5.8^{h}$ \\
\hline PET07 & $1004.6^{a}$ & $315.0^{b}$ & $4.3^{b c}$ & $22.5^{b c}$ & $8.8^{d e f}$ \\
\hline PET08 & $839.8^{a g}$ & $442.6^{a}$ & $5.3^{b}$ & $16.6^{\text {cde }}$ & $11.1^{b c d}$ \\
\hline JMM09 & $556.2^{d e}$ & $264.9^{b c d}$ & $3.2^{\text {cde }}$ & $15.5^{\text {cde }}$ & $6.0^{g h}$ \\
\hline JMM10 & $770.8^{b c}$ & $199.6^{e f g}$ & $2.4^{d e}$ & $15.2^{\text {cde }}$ & $8.7^{\text {def }}$ \\
\hline PET11 & $534.3^{d e f}$ & $323.0^{b}$ & $3.3^{\text {cde }}$ & $20.5^{b c d}$ & $9.9^{\text {cde }}$ \\
\hline CHE12 & $577.5^{d}$ & $194.4^{e f g}$ & $2.4^{d e}$ & $18.2^{\text {cde }}$ & $10.8^{b c d}$ \\
\hline FCP13 & $768.5^{b c}$ & $219.8^{\text {cde }}$ & $3.4^{\text {cde }}$ & $21.4^{b c d}$ & $8.6^{e f g}$ \\
\hline HAL14 & $407.9^{e f}$ & $212.0^{\text {cde }}$ & $2.7^{d e}$ & $17.5^{c d e}$ & $6.4^{f g h}$ \\
\hline PET15 & $219.2^{g}$ & $125.5^{g}$ & $2.3^{e}$ & $4.4^{f}$ & $2.8^{i}$ \\
\hline CV (\%) & 17.18 & 21.15 & 26.08 & 25.76 & 18.67 \\
\hline
\end{tabular}

\section{Determinación del contenido mineral}

El análisis de varianza para el contenido mineral en las semillas detecto diferencias significativas ( $p \leq 0.05$ ) en todos los elementos cuantificados en las colectas (Tabla 5). La colecta OXC04 fue la de mayor contenido de $\mathrm{Ca}, \mathrm{Mn}, \mathrm{Fe}$ y $\mathrm{Zn}$. Mientras que la colecta PET15 fue la que tuvo las concentraciones más bajas en los minerales determinados. La concentración de K fluctuó entre 219.2 y 1004.6 $\mathrm{mg} \mathrm{kg}^{-1}$ con promedio de $631.17 \mathrm{mg} \mathrm{kg}^{-1}$; mientras que el Ca osciló entre 125.5 y $468.3 \mathrm{mg} \mathrm{kg}^{-1}$. En tanto que el Mn estuvo en una concentración de 2.3 a $8.9 \mathrm{mg} \mathrm{kg}^{-1}$. Para el contenido de $\mathrm{Fe}$, las colectas OXC04, OXC05 y PET07 tuvieron las mayores concentraciones con $34.5,27.5$ y $22.5 \mathrm{mg} \mathrm{kg}^{-1}$, respectivamente. Mientras que el contenido de $\mathrm{Zn}$ oscilo entre 2.8 y $15.0 \mathrm{mg} \mathrm{kg}^{-1}$, presentando los mayores valores las colectas HEC02 y HEC03 (Tabla 5).

\section{DISCUSIÓN}

\section{Diversidad fenotípica}

Los resultados indican que las semillas de frijol caupí presentan diversidad fenotípica, predominando semillas de forma ovoide con textura suave. Lo cual es similar a lo reportado por Doumbia et al. (2013) al caracterizar colectas de frijol caupí, pero difiere con Stoilova y Pereira (2013) quienes reportan $44 \%$ de semillas con texturas suaves y $56 \%$ ásperas. Esto se puede deber a que la región de colecta está fuertemente influenciada por el mercado local y los productores siembran las semillas que mejor aceptación tienen. Al respecto, se sabe que los consumidores prefieren el frijol caupí con textura áspera, porque absorbe agua con mayor rapidez y presentan menor tiempo de cocción; además, es más fácil quitar la cubierta de semilla de textura rugosa cuando se procesan los granos en algunos productos alimenticios (Muñoz-Velázquez et al. 2009, Boukar et al. 2013).

El color de la semilla es una característica ligada al consumidor, que influye sobre la selección de los genotipos que cultivan los productores (Dugje et al. 2009). Al respecto se encontró que el $80 \%$ de las colectas tuvieron semilla de color negro, debido que en el sureste de México se prefiere consumir frijoles con este color (Rodríguez et al. 2010), pero también se encontraron de color crema y blanco como las colectas HEC03 y PET08, que puede ser una alternativa para mercados en el centro y norte de México, así como para mercados internacionales, donde se prefiera consumir frijol caupí de color claro (Timko y Singh, 2008). Al respecto, Martínez-Castillo et al. (2008) mencionan que la preferencia de los consumidores por algunos colores, pone en riesgo de erosión genética a la especie, ya que provoca que algunos genotipos se pierdan por desplazamiento de materiales con atributos deseables, reduciendo la diversidad y por lo tanto, de la especie, por lo que es importante conservar genotipos con genes que pueden ser utilizados en el mejoramiento de la especie.

El peso individual de las semillas del frijol caupí oscila entre 0.08 y $0.32 \mathrm{~g}$ (Kaptso et al. 2008), por lo que el $93 \%$ de las colectas están dentro de este rango, mientras que el $7 \%$ restante está por debajo 
del valor mínimo. Sobre lo mismo, Ogle et al. (1987) clasificaron las variedades de frijol caupí en cuatro categorías con base en el peso de 100 semillas, considerando como semillas pequeñas aquellas con peso menor a $15 \mathrm{~g}$, semillas medianas de $15.1 \mathrm{a}$ $20 \mathrm{~g}$, semillas grandes de 20.1 a $25 \mathrm{~g}$ y semillas muy grandes con peso superior a $25 \mathrm{~g}$. De acuerdo con la clasificación anterior el $53 \%$ (ocho colectas) se clasifican como semillas pequeñas, el $40 \%$ (seis colectas) como semillas medianas y $7 \%$ (una colecta) como semillas grandes. Esta variabilidad de tamaños en semillas de frijol caupí se puede deber a que no es una característica importante para el consumidor mexicano al momento de seleccionar el tipo de frijol, dado que las características de mayor influencia son el color, olor después de cocinarse, sabor y tiempo de cocción (Guzmán et al. 2009, Rodriguez et al. 2010).

Las colectas estudiadas tienen longitud entre 6.26 y $9.56 \mathrm{~mm}$, lo que coincide con lo reportado por Kabas et al. (2007) quienes reportan valores entre 5.5 y $9.0 \mathrm{~mm}$, presentando la colecta HEC09 la mayor longitud. El tamaño de la semilla de frijol caupí es un criterio que puede determinar el uso final en el mercado, por ejemplo las variedades con semillas grandes son preferidas para enlatado (Henshaw 2008). Por otra parte es importante para el productor, debido a que semillas de mayor tamaño tienen mayor rendimiento. En cuanto al diámetro medio geométrico y diámetro medio aritmético la colecta HEC03 obtuvo los mayores valores, al ser la colecta con semillas de mayor tamaño. Al respecto Rojas-Barahona y Aristizábal (2011) mencionan que la humedad genera un dilatamiento de las estructuras celulares aumentando el tamaño del mismo. Para la esfericidad de las semillas, todas las colectas presentan valores cercanos a los reportados para la geometría de un cubo $(0.80 \%)$ y distantes de los valores de geometría cilíndrica (0.87) y esférica $(1.0 \%)$ (Bayram 2005), rasgo que es importante para los equipos de clasificación y control de calidad en la industria agroalimentaria (Vizcarra et al. 2017).

El grosor de la testa es una característica que puede ayudar en el manejo de postcosecha de las semillas, especialmente para el tiempo de conservación, debido a que las variedades con testa del- gada se agrietan y se rompen fácilmente, mientras que las semillas con testa más gruesa presentan mayor resistencia a ataques por plagas (Ayala et al. 2006), por lo tanto, la colecta PET15 que obtuvo el mayor grosor de testa, puede ser una alternativa para su conservación por mayor tiempo. Por otra parte, las colectas JMM10 y CHE12 fueron las de menor grosor de testa, por lo que pueden ser preferible para el consumidor debido a la relación que tiene esta característica con el tiempo de cocción.

Los cuatro grupos definidos muestran que la variación no está ligado al lugar de procedencia de la semilla, ya que en cada grupo se encuentran colectas de los diferentes estados de la península, lo que puede deberse a que los productores por lo general intercambian semillas entre ellos o en otros casos compran las semillas en mercados locales o ferias de semillas (Dzib-Aguilar et al. 2016). También los productores acostumbran sembrar dos o más tipos de semillas por ciclo del cultivo. La colecta PET15 y HEC03 se agruparon solas en el dendograma, debido a que presentaron características diferentes de las otras colectas, la colecta PET15 tiene la semilla de menor peso y longitud, mayor grosor de testa, de color rojo y la única de forma romboide. Mientras que la colecta HAC03 es la de mayor peso, anchura y espesor, además es la única colecta de color crema. Por otro lado, debido a que los grupos fueron formados en base con las características fenotípicas, y no con base en los sitios de origen, es un indicativo de que los genotipos comparten características muy cercanas aumentando así la diversidad genética de la especie en la región.

\section{Determinación del contenido mineral}

El análisis estadístico presenta diferencias significativas $(p \leq 0.05)$ entre colectas, lo que confirma la diversidad genotípica. Entre los materiales analizados, las colectas con semillas grandes presentaron mayor contenido de minerales, debido a su mayor capacidad para almacenar nutrientes (OrtegaDavid et al. 2010). Pero, los resultados obtenidos son inferiores a lo reportado en otros estudios (Timko y Singh 2008, Boukar et al. 2011, GuillénMolina et al. 2016). La variabilidad en la con- 
centración de minerales de las semillas locales de frijol caupí, puede asociarse con factores como el genotipo, características morfológicas, condiciones edafoclimáticas y las prácticas culturales que los agricultores realizan durante el ciclo de cultivo (Astudillo y Blair 2008, Chavez-Mendoza y Sanchez 2017), las diferencias de las colectas también puede deberse al método de análisis (Espinoza-García et al. 2016).

La baja concentración de Ca en las colectas, posiblemente se deba a la dependencia que tiene con compuestos como el oxalato y en menor medida al contenido de fítato, que como consecuencia no permite que el elemento tenga buena biodisponibilidad para el consumidor (Moraghan et al. 2006). El contenido de Mn es inferior a lo reportado por Carvalho et al. (2012). Para el contenido de Fe, la colecta OXC04 tuvo la mayor concentración con 34.5 $\mathrm{mg} \mathrm{kg}^{-1}$, los cuales no son suficientes para satisfacer los requerimientos de la población que consume esta especie como alimento básico; al respecto Timko y Singh (2008) mencionan que se requieren concentraciones de 48 a $79 \mathrm{mg} \mathrm{kg}^{-1}$ de Fe en las semillas de frijol caupí para considerarse como alimento autosuficiente. Pero la concentración de la colecta OXC04 es similar a algunos genotipos de frijol común (Phaseolus vulgaris) que se consumen en México (Chavez-Mendoza y Sanchez 2017), por lo que puede ser una alternativa para el consumidor.

El contenido de zinc es menor en un 224 y $227.3 \%$ a los reportados en variedades criollas colec- tados por De Paula et al. (2016) y Muranaka et al. (2015). Al respecto se sabe que la concentración de $\mathrm{Zn}$ en las semillas depende de la disponibilidad del elemento en el suelo y está relacionado con el $\mathrm{pH}$. Cuando el $\mathrm{pH}$ del suelo es superior a 7.0 el Zn disponible disminuye de 30 a 45\% (Gupta et al. 2016); al respecto en la Península de Yucatán los suelos presentan pH superior a 6.5 (Borges-Gómez et al. 2008, Medina et al. 2009), lo que puede estar correlacionado con la deficiencia de $\mathrm{Zn}$ en las semillas.

\section{CONCLUSIONES}

Las colectas de frijol Caupí estudiadas, tienen amplia diversidad fenotípica. Las variables forma, textura de la testa, color de semilla, peso de semilla, peso de 100 semillas, longitud, ancho, espesor de semilla, diámetro medio geométrico, diámetro aritmético, esfericidad, relación ancho-longitud de la semilla y grosor de la testa, contribuyeron a la diversidad morfológica de las colectas. La diversidad fenotípica del frijol caupí está distribuida en cuatro grupos principales determinados por las características morfológicas de la semilla y no por su origen geográfico. El frijol Caupí de la Península de Yucatán, tiene semillas pequeñas de color negro, con variabilidad en el contenido mineral entre las colectas. Las semillas de mayor tamaño presentaron mayor contenido mineral, presentando los mayores contenidos las colectas OXC04 y PET07.

\section{LITERATURA CITADA}

Altuntaş E, Yıldız M (2007) Effect of moisture content on some physical and mechanical properties of faba bean (Vicia faba L.) grains. Journal of Food Engineering 78: 174-183.

Arias L, Jarvis D, Williams D, Latournerie L, Márquez F, Castillo F, et al. (2004) Conservación in situ de la biodiversidad de las variedades locales en la milpa de Yucatán, México. En: Chávez JL, Tuxill J, Jarvis DI (eds): Manejo de la diversidad de los cultivos en los agroecosistemas tradicionales. Instituto Internacional de Recursos Fitogenéticos, Cali, Colombia. pp: 36-46.

Astudillo C, Blair MW (2008) Contenido de hierro y zinc en la semilla y su respuesta al nivel de fertilización con fósforo en 40 variedades de frijol colombianas. Agronomía Colombiana 26: 471-476.

Ayala GOJ, Pichardo GJM, Estrada GJA, Carrillo SJA, Hernández LA (2006) Rendimiento y calidad de semilla del frijol ayocote en el Valle de México. Agricultura Técnica en México 32: 313-321. 
Bayram M (2005) Determination of the sphericity of granular food materials. Journal Food Engineering 68: 385390.

Borges-Gómez L, Soria-Fregoso M, Casanova-Villarreal V, Villanueva-Cohuo E, Pereyda-Pérez G (2008) Correlación y calibración del análisis de fósforo en suelos de Yucatán, México, para el cultivo de chile habanero. Agrociencia 42: 21-27.

Boukar O, Bhattacharjee R, Fatokun C, Kumar PL, Gueye B (2013) Cowpea. In: Singh M, Upadhyaya HD, Singh IB (ed). Genetic and genomic resources of grain legume improvement. Elsevier Inc. London, England. pp: 137-156.

Boukar O, Massawe F, Muranaka S, Franco J, Maziya-Dixon B, Singh B, et al. (2011) Evaluation of cowpea germplasm lines for protein and mineral concentrations in grains. Plant Genetic Resources 9: 515-522.

Cardoso P, Mateus TC, Velu G, Singh RP, Santos JP, Carvalho ML, et al. (2018) Localization and distribution of Zn and $\mathrm{Fe}$ in grains of biofortified bread wheat lines through micro-and triaxial-X-ray fluorescence spectrometry. Spectrochimica Acta Part B: Atomic Spectroscopy 141: 70-79.

Carvalho AFU, De Sousa NM, Farias DF, Da Rocha-Bezerra LCB, Da Silva RMP, Viana MP, et al. (2012) Nutritional ranking of 30 Brazilian genotypes of cowpeas including determination of antioxidant capacity and vitamins. Journal of Food Composition and Analysis 26: 81-88.

Carvalho M, Lino-Neto T, Rosa E, Carnide V (2017) Cowpea: a legume crop for a challenging environment. Journal Sciencie Food Agricultural 97: 4273-4284.

Cetin M (2007) Physical properties of barbunia bean (Phaseolus vulgaris L. cv. "Barbunia") seed. Journal of Food Engineering 80: 353-358.

Coulibaly O, Lowenberg-DeBoer J (2002) The economics of cowpea in West Africa. In Proceedings, Third World Cowpea Conference, Challenges and opportunities for enhancing sustainable cowpea production. Ibadan: IITA. Nigeria. pp: 351-366.

Chavez-Mendoza C, Sanchez E (2017) Bioactive compounds from mexican varieties of the common bean (Phaseolus vulgaris): Implications for health. Molecules 22 1360. Doi:10.3390/molecules22081360.

De Paula CD, Jarma S, Aramendiz H (2016) Caracterización nutricional y sensorial de frijol caupí (Vigna unguiculata (L.) Walp.). Agronomía Colombiana 34: S1131-S1134.

Demooy B, Demooy C (1990) Evaluation of cooking time and quality of seven diverse cowpea (Vigna unguiculata (L.) Walp.) varieties. International journal of food science \& technology 25: 209-212.

Deng GF, Lin X, Xu XR, Gao LL, Xie JF, Li HB (2013) Antioxidant capacities and total phenolic contents of 56 vegetables. Journal of Functional Foods 5: 260-266.

Doumbia IZ, Akromah R, Asibuo JY (2013) Comparative study of cowpea germplasms diversity from Ghana and Mali using morphological characteristics. Journal Plant Breeding and Genetics 1: 139-147.

Dugje IY, Omoigui LO, Ekeleme F, Kamara AY, Ajeigbe H (2009) Farmers guide to cowpea production in West Africa. International Institute of Tropical Agriculture. Ibadan, Nigeria, 19p.

Dzib-Aguilar LA, Ortega-Paczka R, Segura-Correa JC (2016) Conservación in situ y mejoramiento participativo de maíces criollos en la península de Yucatán. Tropical and Subtropical Agroecosystems 19: 51-59.

Espinoza-García N, Martínez-Martínez R, Chávez-Servia JL, Vera-Guzmán AM, Carrillo-Rodríguez JC, HerediaGarcía E, Velasco-Velasco VA (2016) Contenido de minerales en semilla de poblaciones nativas de frijol común (Phaseolus vulgaris L.). Revista Fitotecnia Mexicana 39: 215-223. 
Fery RL (2002) New opportunities in Vigna. In: Janick J, Whipkey A (eds) Trends in new crops and new uses. ASHS. Alexandria. VA. EE. UU. pp. 424-428.

García-Hernández JL, Valdez-Cepeda RD, Avila-Serrano NY, Murillo-Amador B, Nieto-Garibay A, MagallanesQuintanar R, et al. (2005) Preliminary compositional nutrient diagnosis norms for cowpea (Vigna unguiculata (L.) Walp.) grown on desert calcareous soil. Plant and Soil 271: 297-307.

Ghalmi N, Malice M, Jacquemin JM, Ounane SM, Mekliche L, Baudoin JP (2010) Morphological and molecular diversity within Algerian cowpea (Vigna unguiculata (L.) Walp.) landraces. Genetic Resources and Crop Evolution 57: 371-386.

Guillén-Molina M, Márquez-Quiroz C, De la Cruz-Lázaro E, Velázquez-Martínez JR, Soto-Parra JM, GarcíaCarrillo M, et al. (2016) Biofortificación de frijol caupí (Vigna unguiculata L. Walp) con hierro y zinc. Revista Mexicana de Ciencias Agrícolas 17: 3427-3438.

Goncalves A, Goufo P, Barros A, Dominguez-Perles R, Trindade H, Rosa EA, et al. (2016) Cowpea (Vigna unguiculata $\mathrm{L}$. Walp), a renewed multipurpose crop for a more sustainable agri-food system: nutritional advantages and constraints. Journal Science Food Agricultural 96: 2941-2951.

Gupta N, Ram H, Kumar B (2016) Mechanism of zinc absorption in plants: Uptake, transport, translocation and accumulation. Reviews in Environmental Science and Bio/Technology 15: 89-109.

Guzmán IT, Almanza EA, Acosta-Gallegos JA, Guzmán-Maldonado SH (2009) Estudio comparativo de características de calidad entre genotipos de frijol de grano negro. Agricultura Técnica en México 35: 449-457.

Henshaw $F$ (2008) Varietal differences in physical characteristics and proximate composition of cowpea (Vigna unguiculata). World Journal of Agricultural Sciences 4: 302-306.

IBPGR (1983) Cowpea descriptors. International Board for Plant Genetic Resources. Rome, Italy. 30p

Kabas O, Yilmaz E, Ozmerzi A, Akinci I (2007) Some physical and nutritional properties of cowpea seed (Vigna sinensis L.). Journal of Food Engineering 79: 1405-1409.

Kaiser HF (1960) The application of electronic computers to factor analysis. Educational and psychological measurement 20: 141-151.

Kaptso KG, Njintang YN, Komnek AE, Hounhouigan J, Scher J, Mbofung CMF (2008) Physical properties and rehydration kinetics of two varieties of cowpea (Vigna unguiculata) and bambara groundnuts (Voandzeia subterranea) seeds. Journal of Food Engineering 86: 91-99.

Lagunes-Espinoza LdC, Gallardo-López F, Becerril-Hernández H, Bolaños-Aguilar E (2008) Diversidad cultivada y sistema de manejo de Phaseolus vulgaris y Vigna unguiculata en la región de la Chontalpa, Tabasco. Revista Chapingo. Serie horticultura 14: 13-21.

Latournerie-Moreno L, Tuxill J, Moo EY, Reyes LA, Alejo JC, Jarvis DI (2006) Traditional Maize Storage Methods of Mayan Farmers in Yucatan, Mexico: Implications for Seed Selection and Crop Diversity. Biodiversity \& Conservation 15: 1771-1795.

Mafakheri K, Bihamta MR, Abbasi AR, Tejada-Moral M (2017) Assessment of genetic diversity in cowpea (Vigna unguiculata L.) germplasm using morphological and molecular characterisation. Cogent Food \& Agriculture 3: $1-20$

Márquez-Quiroz C, De la Cruz-Lázaro E, Osorio-Osorio R, Sánchez-Chávez E (2015) Biofortification of cowpea beans with iron: iron's influence on mineral content and yield. Journal of soil science and plant nutrition 15: 839-847. 
Martínez-Castillo J, Colunga-GarcíaMarín P, Zizumbo-Villarreal D (2008) Genetic erosion and in situ conservation of Lima bean (Phaseolus lunatus L.) landraces in its Mesoamerican diversity center. Genetic Resources and Crop Evolution 55: 1065-1077.

Medina JC, Volke-Haller V, Spínola AG, Ríos JMG, Cruz MJS, FloresvJIC (2009) Propiedades químicas de un Luvisol después de la conversión del bosque a la agricultura en Campeche, México. Agronomía Mesoamericana 20: 217-235

Menssen M, Linde M, Otunga-Omondi E, Abukutsa-Onyango M, Dinssa FF, Winkelmann T (2017) Genetic and morphological diversity of cowpea (Vigna unguiculata (L.) Walp.) entries from East Africa. Scientia Horticulturae 226: 268-276.

Moraghan JT, Etchevers JD, Padilla J (2006) Contrasting accumulations of calcium and magnesium in seed coats and embryos of common bean and soybean. Food Chemistry 95: 554-561.

Muñoz-Velázquez EE, Rubio-Hernández D, Bernal-Lugo I, Garza-García R, Jacinto-Hernández C (2009) Caracterización de genotipos nativos de frijol del estado de Hidalgo, con base a calidad del grano. Agricultura técnica en México 35: 429-438.

Muranaka S, Shono M, Myoda T, Takeuchi J, Franco J, Nakazawa Y, et al. (2015) Genetic diversity of physical, nutritional and functional properties of cowpea grain and relationships among the traits. Plant Genetic Resources 14: 67-76.

Ogle W, Witcher W, Barnett OW (1987) Descriptors for the southern peas of South Carolina. Bulletin/South Carolina Agricultural Experiment Station. No. 659. USA. 23p.

Ortega-David E, Rodriguez A, David A, Zamora-Burbano Á (2010) Caracterización de semillas de lupino (Lupinus mutabilis) sembrado en los Andes de Colombia. Acta Agronómica 58: 111-118.

Rodriguez LG, Salazar JAG, Rebollar S, Contreras ACC (2010) Preferencias del consumidor de frijol (Phaseolus vulgaris L.) en México: factores y características que influyen en la decisión de compra diferenciada por tipo y variedad. Paradigma Económico 2: 121-145.

Rojas-Barahona Á, Aristizábal TI (2011) Efecto del contenido de humedad sobre propiedades físicas de la semilla de Vitabosa (Mucuna deeringiana). Revista Facultad Nacional de Agronomía Medellín 64: 5961-5971.

SAS (2009) What's New in SAS ${ }^{\circledR}$ 9.2. SAS Institute Inc. Cary, North Carolina, USA. 84p.

Segura-Campos M, Ruiz-Ruiz J, Chel-Guerrero L, Betancur-Ancona D (2013) Antioxidant activity of Vigna unguiculata L. walp and hard-to-cook Phaseolus vulgaris L. protein hydrolysates. CyTA - Journal of Food 11: 208-215.

SIAP (2017) Anuario estadístico de la producción agrícola - producción agrícola 2017. Servicio de Información Agrícola y Pecuaria. https://nube.siap.gob.mx/cierreagricola/. Fecha de consulta: 10 octubre de 2018.

Smýkal P, Coyne CJ, Ambrose MJ, Maxted N, Schaefer H, Blair MW, et al. (2015) Legume crops phylogeny and genetic diversity for science and breeding. Critical Reviews in Plant Sciences 34: 43-104.

SPSS (2016) Statistics para Windows, Versión 24.0. Armonk, NY: IBM Corp. IBM Corp.

Stoilova T, Pereira G (2013) Assessment of the genetic diversity in a germplasm collection of cowpea (Vigna unguiculata (L.) Walp.) using morphological traits. African Journal of Agricultural Research 8: 208-215.

Timko MP, Singh BB (2008) Cowpea a multifunctional legume. In: More PM, Ming R (Eds.) Genomics of tropical crops plants. Springer. New York, USA. pp: 227-258. 
Vara-Sánchez I, Padilla MC (2013) Biodiversidad cultivada: una cuestión de coevolución y transdisciplinariedad. Revista Ecosistemas 22: 5-9.

Vizcarra TA, Huallpartupa DJR, Buleje EAV, Ramos RP (2017) Caracterización física de semillas de maíz (Zea mayz) sembrado en Andahuaylas Perú. Ciencia \& Desarrollo 18: 56-62.

Wamalwa EN, Muoma J, Wekesa C (2016) Genetic diversity of cowpea (Vigna unguiculata (L.) Walp.) accession in Kenya gene bank based on simple sequence repeat markers. International Journal Genomics Article ID 8956412 Doi: 10.1155/2016/8956412.

Xiong H, Shi A, Mou B, Qin J, Motes D, Lu W, Wu D (2016) Genetic Diversity and Population Structure of Cowpea (Vigna unguiculata L. Walp). PLoS ONE 11(8): e0160941. doi:10.1371/journal.pone.0160941. 
